\title{
Adomian Decomposition Method with Modified Bernstein Polynomials for Solving Nonlinear Fredholm and Volterra Integral Equations
}

\author{
Mohammad Almousa \\ Department of Mathematics, Faculty of Arts and Science, Amman Arab University, Jordan
}

Received February 8, 2020; Revised March 23, 2020; Accepted March 28, 2020

Copyright $\bigcirc 2020$ by authors, all rights reserved. Authors agree that this article remains permanently open access under the terms of the Creative Commons Attribution License 4.0 International License

\begin{abstract}
Many different problems in mathematics, physics, engineering can be expressed in the form of integral equations. Among these are diffraction problems, population growth, heat transfer, particle transport problems, electrical engineering, elasticity, control, elastic waves, diffusion problems, quantum mechanics, heat radiation, electrostatics and contact problems. Therefore, the solutions which are obtained by the mathematical methods play an important role in these fields. The most two basic types of integral equations are called Fredholm (FIEs) and Volterra (VIEs). In many instances, the ordinary and partial differential equations can be converted into Fredhom and Volterra integral equations that are solved more effectively. We aim through this research to present an improved Adomian decomposition method based on modified Bernstein polynomials (ADM-MBP) to solve nonlinear integral equations of the second kind. We introduced efficient method, constructed on modified Bernstein polynomials. The formulation is developed to solve nonlinear Fredholm and Volterra integral equations of second kind. This method is tested for some examples from nonlinear integral equations. Maple software was used to obtain the solutions of these examples. The results demonstrate reliability of the proposed method. Generally, the proposed method is very convenient to apply to find the solutions of Fredholm and Volterra integral equations of second kind.
\end{abstract}

Keywords Adomian Decomposition Method (ADM), Modified Bernstein Polynomials (MBP), Fredholm Integral Equations, Volterra Integral Equations.

\section{Introduction}

Fredholm and Volterra family of integral equations play an important role in mathematics, physics, engineering and others. Therefore, many techniques and numerical methods were used to solve these types of equations. Among these are optimal homotopy asymptotic method $[1,2]$, homotopy perturbation method [3, 4], homotopy analysis method [5], Adomian Decomposition Method [6], barycentric interpolation collocation methods [7], quadrature method based on multivariate Bernstein polynomials [8], integral mean value theorem [9] and Newton-Kantorovich-quadrature method [10]. Other studies can be found on [11-14].

ADM was firstly introduced by G. Adomian [15] and applied it to solve the nonlinear problems in various fields (see [16-18]). Recently, this classical method is modified and developed to improve the accuracy of the results. Hosseini [19] proposed a new improved ADM via Chebyshev polynomials for solving linear and non-linear models. Wazwaz [20] presented the modified ADM to solve linear and nonlinear operators. Song and Wang [21] established a new improved $\mathrm{ADM}$ and applied it to fractional differential equations. Qasim and AL-Rawi [22] used Bernstein polynomials to modify the ADM to solve differential equations. The ADM is improved with orthogonal polynomials [23] and with Laguerre polynomials [24] for solving differential equation. In addition, several improvements were also considered on ADM (see [25- 28]).

The main objective of this research is to introduce a new analytical treatment for nonlinear Fredholm and Volterra integral equations using improved ADM-MBP.

\section{Definitions and Basic Concepts}

To introduce our research we will need the following basic definitions, concepts and results of integral equations and Bernstein polynomials. 


\section{Definition 2.1}

The integral equation in $\mathrm{g}(\mathrm{x})$ is of the form:

$$
\mathrm{g}(\mathrm{x})=\mathrm{f}(\mathrm{x})+\lambda \int_{u(x)}^{h(x)} \mathrm{k}(\mathrm{x}, \mathrm{t}) \mathrm{g}(\mathrm{t}) \mathrm{dt},
$$

where $u(x)$ and $h(x)$ may be both fixed, variables, or mixed, $\lambda$ is a constant, $k(x, t)$ is the kernel, $f(x)$ is given function and $g(x)$ is unknown. If $u(x)$ and $h(x)$ are fixed, then it is called a Fredholm. If at least one limit is a variable, then it is called a Volterra.

\section{Definition 2.2}

The Bernstein basis polynomials of degree $m$ are defined by

$$
\mathrm{B}_{\mathrm{i}, \mathrm{m}}(\mathrm{x})=\left(\begin{array}{c}
\mathrm{m} \\
\mathrm{i}
\end{array}\right) \mathrm{x}^{\mathrm{i}}(1-\mathrm{x})^{\mathrm{m}-\mathrm{i}}
$$

where $0 \leq \mathrm{i} \leq \mathrm{m}, \quad 0 \leq \mathrm{x} \leq 1$, and $\left(\begin{array}{c}\mathrm{m} \\ \mathrm{i}\end{array}\right)=\frac{\mathrm{m} !}{\mathrm{i} !(\mathrm{m}-\mathrm{i}) !}$.

\section{Definition 2.3}

A linear combination of Bernstein basis polynomials

$$
\mathrm{B}_{\mathrm{m}}(\mathrm{x})=\sum_{\mathrm{i}=0}^{\mathrm{m}} \mathrm{B}_{\mathrm{i}, \mathrm{m}}(\mathrm{x}) \beta_{\mathrm{i}}
$$

is called the Bernstein polynomials, where $\beta_{i}$ are the Bernstein coefficients.

Definition 2.4

The $m$ th Bernstein polynomial for $f(x)$ defined by

$$
B_{m}(f)=\sum_{i=0}^{m} B_{i, m}(x) f\left(\frac{i}{m}\right),
$$

Note that here for each function $f:[0,1] \rightarrow R$, we have

$$
\lim _{\mathrm{m} \rightarrow \infty} B_{m}(f)=f(x) .
$$

\section{Remark 2.1}

The derivatives of the $m$ th degree Bernstein polynomials are given by

$$
\frac{d}{d x} B_{i, m}(x)=m\left(B_{i-1, m-1}(x)-B_{i, m-1}(x)\right) .
$$

It should be noted that the $2 k$ th order derivative $\mathrm{f}^{(2 \mathrm{k})}$ has been shown by [29]

$$
\mathrm{B}_{\mathrm{m}}^{\mathrm{f}}(\mathrm{x})=\mathrm{f}(\mathrm{x})+\sum_{\mathrm{a}=2}^{2 \mathrm{k}-1} \frac{\mathrm{f}^{(\mathrm{a})}(\mathrm{x})}{\mathrm{a} ! \mathrm{m}^{\mathrm{a}}} \mathrm{T}_{\mathrm{m}, \mathrm{a}}(\mathrm{x})+\mathrm{O}\left(\frac{1}{\mathrm{~m}^{\mathrm{k}}}\right)
$$

where

$$
\mathrm{T}_{\mathrm{m}, \mathrm{a}}(\mathrm{x})=\sum_{k}(k-m x)^{a}\left(\begin{array}{l}
m \\
k
\end{array}\right) x^{k}(1-x)^{m-k}
$$

\section{Description of the Modified Technique}

In order to apply the new modified technique to nonlinear integral equation, we rewrite Eq. (1) as

$$
\mathrm{g}(\mathrm{x})=\mathrm{f}(\mathrm{x})+\lambda \int_{u(x)}^{h(x)} \mathrm{k}(\mathrm{x}, \mathrm{t}) \mathrm{N}(\mathrm{g}(\mathrm{t})) \mathrm{dt}
$$

where $\mathrm{N}(\mathrm{g}(\mathrm{t}))$ is a nonlinear function.

By Adomian polynomials, $\mathrm{N}(\mathrm{g}(\mathrm{t}))$ is given by

$$
\mathrm{N}(\mathrm{g})=\sum_{n=0}^{\infty} \mathrm{A}_{\mathrm{n}}\left(\mathrm{g}_{1}, \mathrm{~g}_{2}, \ldots, \mathrm{g}_{\mathrm{n}}\right),
$$

where $\mathrm{A}_{\mathrm{n}}=\frac{1}{\mathrm{n} !} \frac{\mathrm{d}^{\mathrm{n}}}{\mathrm{d} \gamma} \mathrm{n}\left[\mathrm{N}\left(\sum_{i=0}^{\infty} \gamma^{\mathrm{i}} \mathrm{g}_{\mathrm{i}}\right)\right], \mathrm{n}=0,1,2, \ldots$

Using Eqs. (4), (6) and (7), the modified Bernstein series can be obtained

$$
\begin{aligned}
& f(x)=\sum_{i=0}^{m}\left(\begin{array}{c}
m \\
i
\end{array}\right) x^{i}(1-x)^{m-i} f\left(\frac{i}{m}\right) \\
& -\sum_{a=2}^{2 k-1} \frac{\left(\frac{d^{a}}{d x^{a}}\right) B_{i, m}(x)}{a ! m^{a}} T_{m, a}(x) .
\end{aligned}
$$

Now, we can obtain the solution $\mathrm{g}(\mathrm{x})$ by

$$
\mathrm{g}(\mathrm{x})=\sum_{j=0}^{\infty} \mathrm{g}_{\mathrm{j}}(\mathrm{x}) \text {. }
$$

Substituting Eqs. (10), (11) and (12) into Eq. (9) gives

$$
\begin{aligned}
& \sum_{j=0}^{\infty} \mathrm{g}_{\mathrm{j}}(\mathrm{x})=\sum_{i=0}^{\mathrm{m}}\left(\begin{array}{c}
\mathrm{m} \\
\mathrm{i}
\end{array}\right) \mathrm{x}^{\mathrm{i}}(1-\mathrm{x})^{\mathrm{m}-\mathrm{i}} \mathrm{f}\left(\frac{\mathrm{i}}{\mathrm{m}}\right) \\
& -\sum_{\mathrm{a}=2}^{2 \mathrm{k}-1} \frac{\left(\frac{\mathrm{d}^{\mathrm{a}}}{\mathrm{d \textrm {x } ^ { \mathrm { a } }}}\right) \mathrm{B}_{\mathrm{i}, \mathrm{m}}(\mathrm{x})}{\mathrm{a} ! \mathrm{m}^{\mathrm{a}}} \mathrm{T}_{\mathrm{m}, \mathrm{a}}(\mathrm{x}) \\
& +\lambda \int_{u(x)}^{h(x)} \mathrm{k}(\mathrm{x}, \mathrm{t}) \mathrm{A}_{\mathrm{n}} \mathrm{dt}
\end{aligned}
$$




$$
\begin{gathered}
\mathrm{g}_{0}(\mathrm{x})=\sum_{i=0}^{\mathrm{m}}\left(\begin{array}{c}
\mathrm{m} \\
\mathrm{i}
\end{array}\right) \mathrm{x}^{\mathrm{i}}(1-\mathrm{x})^{\mathrm{m}-\mathrm{i}} \mathrm{f}\left(\frac{\mathrm{i}}{\mathrm{m}}\right) \\
-\sum_{\mathrm{a}=2}^{2 \mathrm{k}-1} \frac{\left(\frac{\mathrm{d}^{\mathrm{a}}}{\mathrm{dx}^{\mathrm{a}}}\right) \mathrm{B}_{\mathrm{i}, \mathrm{m}}(\mathrm{x})}{\mathrm{a} ! \mathrm{m}^{\mathrm{a}}} \mathrm{T}_{\mathrm{m}, \mathrm{a}}(\mathrm{x}) \\
\mathrm{g}_{\mathrm{n}+1}(\mathrm{x})=\lambda \int_{u(x)}^{h(x)} \mathrm{k}(\mathrm{x}, \mathrm{t}) \mathrm{A}_{\mathrm{n}} \mathrm{dt}, \mathrm{n}=0,1,2, \ldots
\end{gathered}
$$

The ADM-MBP solution can be obtained as

$$
\mathrm{g}_{\mathrm{m}}(\mathrm{x})=\sum_{j=0}^{\infty} \mathrm{g}_{\mathrm{j}}(\mathrm{x})
$$

Theorem 3.1

The series (15) of Eq. (1) converges if $\exists \alpha \in[0,1)$ and $\left\|g_{n+1}\right\|=\alpha\left\|g_{n}\right\|$ such that $\left\|g_{0}\right\|<\infty$.

Proof. Let $S_{n}=g_{1}+g_{2}+\ldots+g_{n}$. For $m \geq n$, we shall prove that $S_{n}$ is a Cauchy sequence

$$
\left\|S_{m}-S_{n}\right\|=\left\|\sum_{i=0}^{m} y i-\sum_{i=0}^{n} y i\right\|=\left\|\sum_{i=n+1}^{m} y_{i}\right\| .
$$

We know that

$$
\begin{gathered}
\left\|S_{m}-S_{n}\right\|=\left\|\left(S_{n+1}-S_{n}\right)+\left(S_{n+2}-S_{n+1}\right)+\ldots+\left(S_{m}-S_{m-1}\right)\right\| \\
\leq\left\|S_{n+1}-S_{n}\right\|+\left\|S_{n+2}-S_{n+1}\right\|+\ldots+\left\|S_{m}-S_{m-1}\right\| \\
\leq \alpha^{n+1}\left\|g_{0}\right\|+\alpha^{n+2}\left\|g_{0}\right\|+\ldots+\alpha^{m}\left\|g_{0}\right\| \\
\leq\left(\alpha^{n+1}+\alpha^{n+2}+\ldots+\alpha^{m}\right)\left\|g_{0}\right\| \\
\leq \alpha^{n+1}\left(1+\alpha+\ldots+\alpha^{m-n-1}\right)\left\|g_{0}\right\| \\
\leq \alpha^{n+1}\left(\frac{1-\alpha^{m-n}}{1-\alpha}\right)\left\|g_{0}\right\| \\
\leq\left(\frac{\alpha^{n+1}}{1-\alpha}\right)\left\|g_{0}\right\| \quad\left(\text { Since } \alpha \in[0,1) \Rightarrow 1-\alpha^{m-n}<1\right)
\end{gathered}
$$

But $\left\|g_{0}\right\|<\infty$, i.e. $\left\|S_{m}-S_{n}\right\| \rightarrow 0$ as $n \rightarrow 0$. So the series converges.

\section{Theorem 3.2}

The maximum absolute error of Eq. (15) can be obtained as

$$
\max _{\forall x \in J}\left|g(x)-\sum_{i=0}^{n} g_{i}(x)\right| \leq\left(\frac{\alpha^{n+1}}{1-\alpha}\right) \max _{\forall x \in J}\left\|g_{0}\right\| .
$$

Proof. From Theorem 3.1 for $m \rightarrow \infty$ then $S_{m} \rightarrow g(x)$, so

$$
\left\|g(x)-S_{n}\right\| \leq\left(\frac{\alpha^{n+1}}{1-\alpha}\right)\left\|g_{0}\right\|
$$

Thus

$$
\max _{\forall x \in J}\left|g(x)-\sum_{i=0}^{n} g_{i}(x)\right| \leq\left(\frac{\alpha^{n+1}}{1-\alpha}\right) \max _{\forall x \in J}\left\|g_{0}\right\| .
$$

\section{Applications}

Some applications of nonlinear integral equations are solved to show the accuracy of the new modified technique.

\section{Application 4.1}

Consider the VIE given by [30]

$$
g(x)=x+\int_{0}^{x} g^{2}(t) d t
$$

with analytical solution $\mathrm{g}(\mathrm{x})=\tan x$. Using ADM then $\mathrm{N}(\mathrm{g})=\mathrm{g}^{2}(\mathrm{t})$ and $f(x)=x$.

Applying the Adomian polynomials to $\mathrm{N}(\mathrm{g})=\mathrm{g}^{2}(\mathrm{t})$, we have

$$
\begin{aligned}
& \mathrm{A}_{0}=\mathrm{N}\left(\mathrm{g}_{0}\right)=\mathrm{g}_{0}^{2}(\mathrm{t}), \\
& \mathrm{A}_{1}=2 \mathrm{~g}_{0}(\mathrm{t}) \mathrm{g}_{1}(\mathrm{t}), \\
& \mathrm{A}_{2}=2 \mathrm{~g}_{0}(\mathrm{t}) \mathrm{g}_{2}(\mathrm{t})+\mathrm{g}_{1}^{2}(\mathrm{t}), \\
& \mathrm{A}_{3}=2 \mathrm{~g}_{0}(\mathrm{t}) \mathrm{g}_{3}(\mathrm{t})+2 \mathrm{~g}_{1}(\mathrm{t}) \mathrm{g}_{2}(\mathrm{t}),
\end{aligned}
$$

and so on. Using Eqs. (11) - (14) when $\mathrm{m}=6$ and $k=2$, we set

$$
\begin{gathered}
\mathrm{g}_{0}(\mathrm{x})=\sum_{i=0}^{6}\left(\begin{array}{l}
6 \\
\mathrm{i}
\end{array}\right) \mathrm{x}^{\mathrm{i}}(1-\mathrm{x})^{6-\mathrm{i}} \mathrm{f}\left(\frac{\mathrm{i}}{6}\right) \\
-\sum_{\mathrm{a}=2}^{3} \frac{\left(\frac{\mathrm{d}^{\mathrm{a}}}{\mathrm{dx^{ \textrm {a } }}}\right) \mathrm{B}_{\mathrm{i}, 6}(\mathrm{x})}{\mathrm{a} ! 6^{\mathrm{a}}} \mathrm{T}_{6, \mathrm{a}}(\mathrm{x}) \\
=\mathrm{x}(1-\mathrm{x})^{5}+5 \mathrm{x}^{2}(1-\mathrm{x})^{4}+10 \mathrm{x}^{3}(1-\mathrm{x})^{3} \\
+10 \mathrm{x}^{4}(1-\mathrm{x})^{2}+5 \mathrm{x}^{5}(1-\mathrm{x})+\mathrm{x}^{6}=x
\end{gathered}
$$




$$
\begin{aligned}
& g_{1}(x)=\int_{0}^{x} A_{0} d t=\frac{1}{3} x^{3} \\
& g_{2}(x)=\int_{0}^{x} A_{1} d t=\frac{2}{15} x^{5} \\
& g_{3}(x)=\int_{0}^{x} A_{2} d t=\frac{17}{315} x^{7} \\
& g_{4}(x)=\int_{0}^{x} A_{3} d t=\frac{62}{2835} x^{9}
\end{aligned}
$$

and so on. This in turn gives

$$
\begin{aligned}
& g_{\mathrm{m}}(\mathrm{x})=\sum_{j=0}^{\infty} \mathrm{g}_{j}(\mathrm{x})=\mathrm{x}+\frac{1}{3} \mathrm{x}^{3}+\frac{2}{15} \mathrm{x}^{5}+\frac{17}{315} \mathrm{x}^{7} \\
& +\frac{62}{2835} \mathrm{x}^{9}+\ldots=\tan (\mathrm{x}) .
\end{aligned}
$$

Table 1 presents the solutions of $\mathrm{g}_{7}(\mathrm{x})$ with $m=6$ and $k=2$.

Table 1. Solutions of Application 1.

\begin{tabular}{|c|c|c|c|}
\hline $\mathrm{x}$ & Exact Solution & $\mathrm{g}_{7}(\mathrm{x})$ & Absolute Error \\
\hline 0 & 0 & 0 & 0 \\
\hline 0.1 & 0.1003346721 & 0.1003346720 & $1.45831214310^{-11}$ \\
\hline 0.2 & 0.2027100355 & 0.2027100356 & $9.00836976910^{-12}$ \\
\hline 0.3 & 0.3093362496 & 0.3093362497 & $8.8296070310^{-12}$ \\
\hline 0.4 & 0.4227932187 & 0.4227932187 & $7.390114010^{-11}$ \\
\hline 0.5 & 0.5463024898 & 0.5463024849 & $4.9616443610^{-9}$ \\
\hline 0.6 & 0.6841368083 & 0.6841366915 & $1.16891344110^{-7}$ \\
\hline 0.7 & 0.8422883805 & 0.8422866677 & $1.71278352910^{-6}$ \\
\hline 0.8 & 1.029638557 & 1.0296206170 & $1.79393152210^{-5}$ \\
\hline 0.9 & 1.260158218 & 1.2600117280 & $1.46490121310^{-4}$ \\
\hline 1.0 & 1.557407725 & 1.5564156070 & $9.9211793610^{-4}$ \\
\hline
\end{tabular}

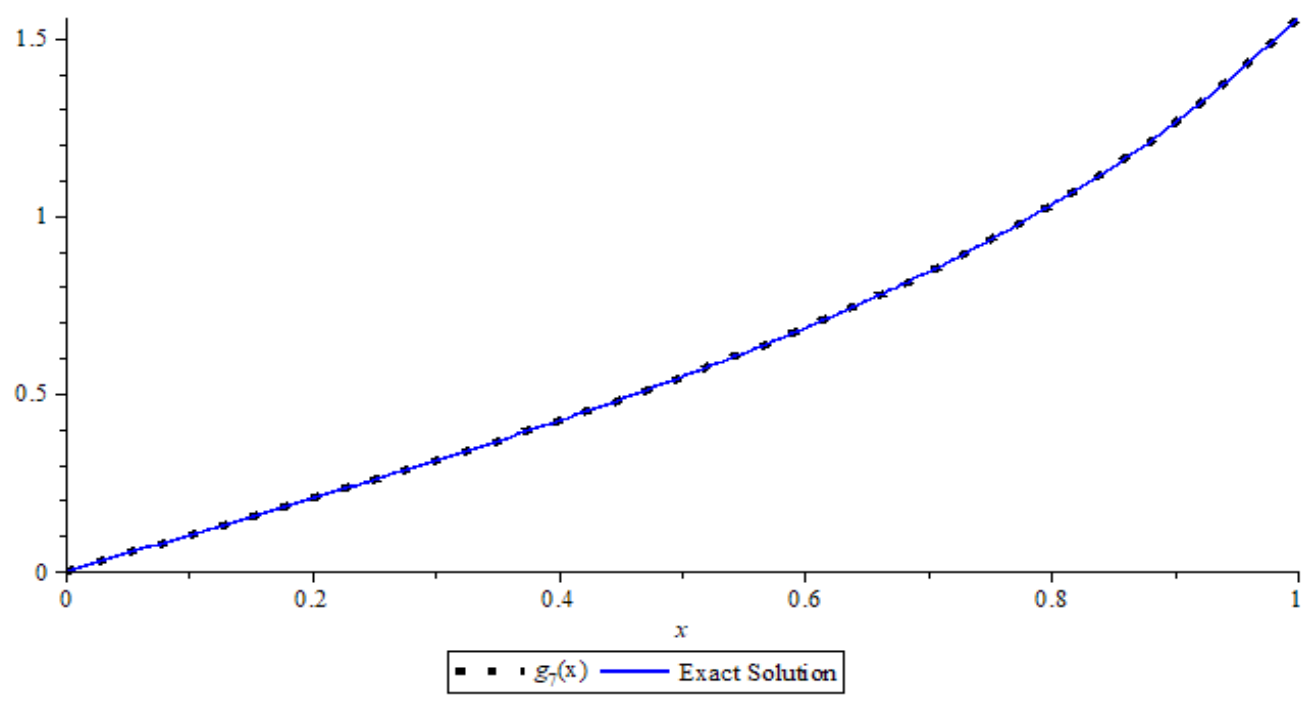

Figure 1. The numerical results obtained by $\mathrm{g}_{7}(\mathrm{x})$ with $\mathrm{m}=6$ and $\mathrm{k}=2$ 


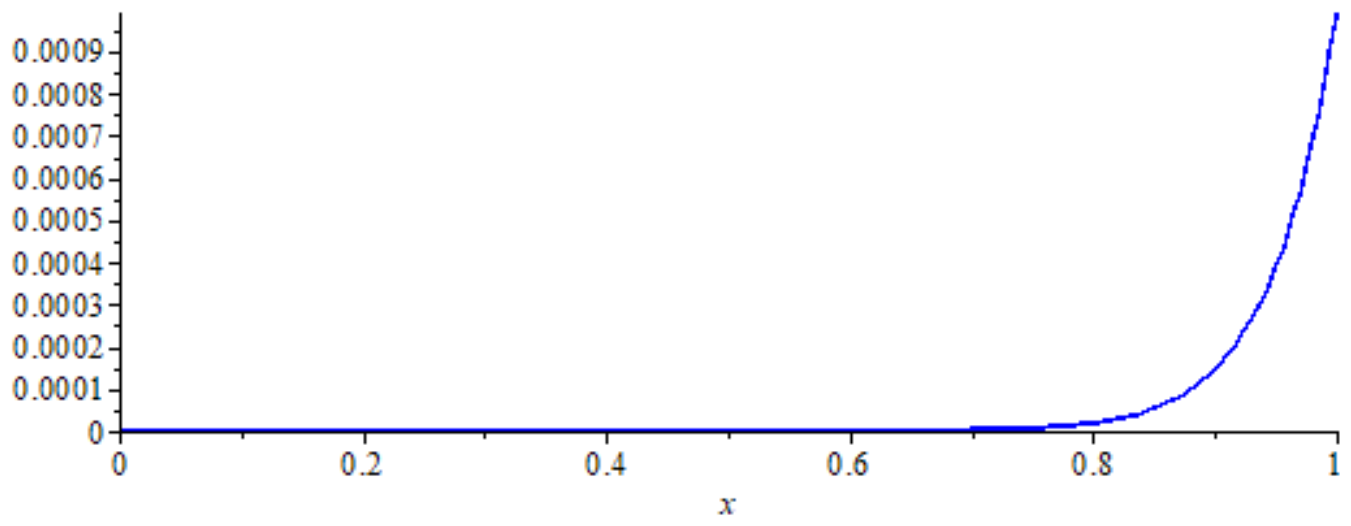

Figure 2. Absolute error between the exact and $\mathrm{g}_{7}(\mathrm{x})$ with $\mathrm{m}=6$ and $\mathrm{k}=2$.

Application 4.2

Consider the FIE given by [30]

$$
g(x)=\frac{5}{6} x+\int_{0}^{1} x t^{2} g^{3}(t) d t
$$

with analytical solution $\mathrm{g}(\mathrm{x})=x$. Using $\mathrm{ADM}$ then $\mathrm{N}(\mathrm{g})=\mathrm{g}^{3}(\mathrm{t})$ and $f(x)=\frac{5}{6} x$. have

Applying the Adomian polynomials to $\mathrm{N}(\mathrm{g})=\mathrm{g}^{3}(\mathrm{t})$, we

$$
\begin{aligned}
& \mathrm{A}_{0}=\mathrm{N}\left(\mathrm{g}_{0}\right)=\mathrm{g}_{0}{ }^{3}(\mathrm{t}), \\
& \mathrm{A}_{1}=3 \mathrm{~g}_{0}^{2}(\mathrm{t}) \mathrm{g}_{1}(\mathrm{t}), \\
& \mathrm{A}_{2}=3 \mathrm{~g}_{0}^{2}(\mathrm{t}) \mathrm{g}_{2}(\mathrm{t})+3 \mathrm{~g}_{1}^{2}(\mathrm{t}) \mathrm{g}_{0}(\mathrm{t}), \\
& \mathrm{A}_{3}=\mathrm{g}_{1}^{3}(\mathrm{t})+6 \mathrm{~g}_{0}(\mathrm{t}) \mathrm{g}_{1}(\mathrm{t}) \mathrm{g}_{2}(\mathrm{t})+3 \mathrm{~g}_{0}^{2}(\mathrm{t}) \mathrm{g}_{3}(\mathrm{t}),
\end{aligned}
$$

and so on. Using Eqs. (11) - (14) when $\mathrm{m}=6$ and $k=2$, we set

$$
\begin{aligned}
& g_{0}(x)=\sum_{i=0}^{6}\left(\begin{array}{l}
6 \\
i
\end{array}\right) x^{i}(1-x)^{6-i} f\left(\frac{i}{6}\right) \\
& -\sum_{a=2}^{3} \frac{\left(\frac{d^{a}}{d x^{a}}\right) B_{i, 6}(x)}{a ! 6^{a}} T_{6, a}(x)
\end{aligned}
$$

$$
\begin{aligned}
& =\frac{5}{6} x(1-x)^{5}+\frac{25}{6} x^{2}(1-x)^{4}+\frac{25}{3} x^{3}(1-x)^{3} \\
& +\frac{25}{3} x^{4}(1-x)^{2}+\frac{25}{9} x^{5}(1-x)+\frac{5}{6} x^{6}=\frac{5}{6} x
\end{aligned}
$$

$g_{1}(x)=\int_{0}^{x} A_{0} d t=\frac{125}{1296} x$

$g_{2}(x)=\int_{0}^{x} A_{1} d t=\frac{3125}{93312} x$

$g_{3}(x)=\int_{0}^{x} A_{2} d t=\frac{78125}{5038848} x$

$g_{4}(x)=\int_{0}^{x} A_{3} d t=\frac{107421875}{13060694016} x$

and so on. This in turn gives

$$
\begin{aligned}
& g_{\mathrm{m}}(\mathrm{x})=\sum_{j=0}^{\infty} \mathrm{g}_{\mathrm{j}}(\mathrm{x})=\frac{5}{6} \mathrm{x}+\frac{125}{1296} \mathrm{x} \\
& +\frac{3125}{93312} \mathrm{x}+\frac{78125}{5038848} \mathrm{x}+\ldots=\mathrm{x} .
\end{aligned}
$$

Table 2 presents the solutions of $\mathrm{g}_{7}(\mathrm{x})$ with $m=6$ and $k=2$. 
Table 2. Solutions of Application 2

\begin{tabular}{|c|c|c|c|}
\hline $\mathrm{x}$ & Exact Solution & $\mathrm{g} 7(\mathrm{x})$ & Absolute Error \\
\hline 0 & 0 & 0 & 0 \\
\hline 0.1 & 0.1 & 0.09963862376 & $3.61376235410^{-4}$ \\
\hline 0.2 & 0.2 & 0.19927724750 & $7.22752470810^{-4}$ \\
\hline 0.3 & 0.3 & 0.29891587130 & $1.08412870610^{-3}$ \\
\hline 0.4 & 0.4 & 0.39855449510 & $1.44550494210^{-3}$ \\
\hline 0.5 & 0.5 & 0.49819311880 & $1.80688117710^{-3}$ \\
\hline 0.6 & 0.6 & 0.59783174260 & $2.16825741210^{-3}$ \\
\hline 0.7 & 0.7 & 0.69747036640 & $2.52963364810^{-3}$ \\
\hline 0.8 & 0.8 & 0.79710899010 & $3.25238611910^{-3}$ \\
\hline 0.9 & 0.9 & 0.89674761390 & $3.61376235410^{-3}$ \\
\hline 1.0 & 1.0 & 0.99638623760 & \\
\hline
\end{tabular}

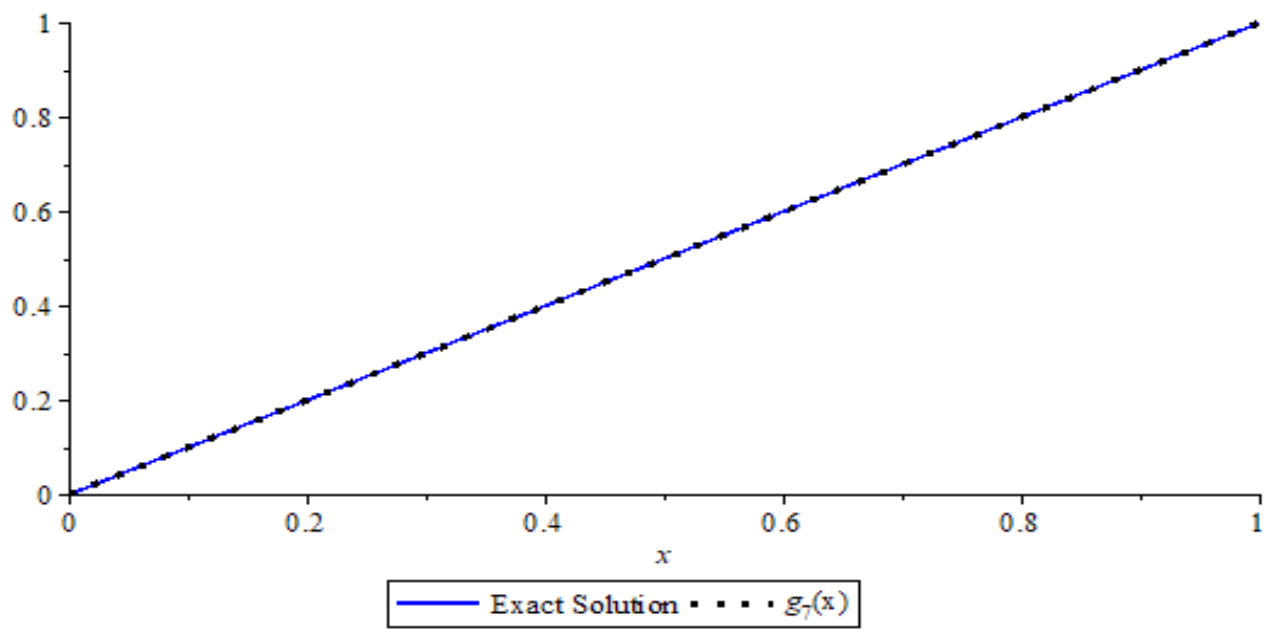

Figure 3. The numerical results obtained by $\mathrm{g}_{7}(\mathrm{x})$ with $\mathrm{m}=6$ and $\mathrm{k}=2$.

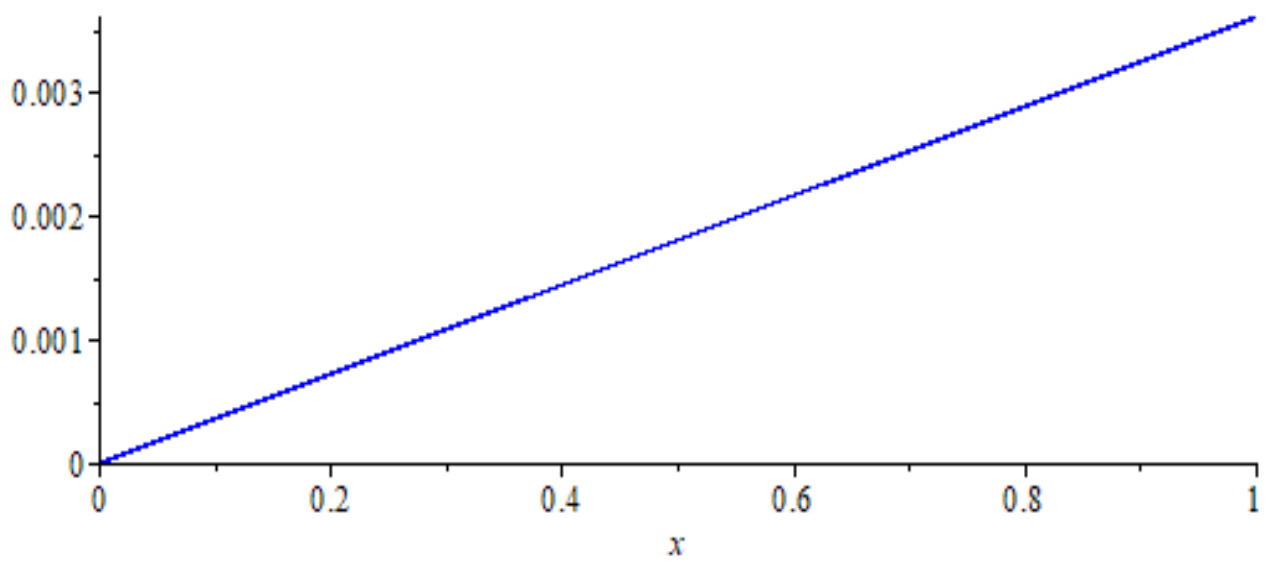

Figure 4. Absolute error between the exact and $\mathrm{g}_{7}(\mathrm{x})$ with $\mathrm{m}=6$ and $\mathrm{k}=2$ 


\section{Conclusions}

We improved ADM based on MBP in order to solve nonlinear Fredholm and Volterra integral equations. This method is tested for some examples from nonlinear integral equations. The results demonstrate reliability of the proposed method.

\section{Acknowledgements}

The author acknowledges the financial support from Amman Arab University, Jordan.

\section{REFERENCES}

[1] M. S. Hashmi, N. Khan and S. Iqbal. Optimal homotopy asymptotic method for solving nonlinear Fredholm integral equations of second kind, Applied Mathematics and Computation, 218 (22), 10982-10989, 2012.

[2] M. Almousa and A. Ismail. Optimal homotopy asymptotic method for solving the linear Fredholm integral equations of the first kind, Abstract and Applied Analysis, (2013); 2013, Article ID 278097, pages.https://doi.org/10.1155/2013/278097.

[3] J. A. Ghorbani and J. Saberi-Nadjafi. Exact solutions for nonlinear integral equations by a modified homotopy perturbation method. Computers \& Mathematics with Applications, 56(4), 1032-1039, 2008.

[4] J. Biazar. Numerical solution for special non-linear Fredholm integral equation by HPM. Applied Mathematics and Computation, 195(2):681-687, 2008.

[5] F. Awawdeh, A. Adawi and S. Al-Shara. A numerical method for solving nonlinear integral equations. International Mathematical Forum, 4(17), 805-8170, 2009.

[6] M. Almousa. The solutions of three dimensional Fredholm integral equations using Adomian decomposition method. AIP Conference Proceedings, 1739(1), 020053-1-020053-6, 2016.

[7] H. Liu, J. Huang, Y. Pan and J. Zhang. Barycentric interpolation collocation methods for solving linear and nonlinear high-dimensional Fredholm integral equations. Journal of Computational and Applied Mathematics, 327(1), $141-154,2018$

[8] Y. Pan, J. Huang and Y. Ma Bernstein series solutions of multidimensional linear and nonlinear Volterra integral equations with fractional order weakly singular kernels. Applied Mathematics and Computation, 347(15), 149-161, 2019.

[9] M. Heydari, Z. Avazzadeh, H. Navabpour and G.B. Loghmani. Numerical solution of Fredholm integral equations of the second kind by using integral mean value theorem II. High dimensional problems. Applied Mathematical Modelling, 37(1-2), 432-442, 2013.

[10] J. Saberi-Nadjafi and M. Heidari. Solving nonlinear integral equations in the Urysohn form by Newton-Kantorovich-quadrature method. Computers \& Mathematics with Applications, 60(7), 2058-2065, 2010.

[11] M. A. Abdou and M. Basseem. Numerical treatments for solving nonlinear mixed integral equation. Alexandria Engineering Journal, 55(4), 3247-3251, 2016.

[12] K. Maleknejad, P. Torabi and R. Mollapourasl. Fixed point method for solving nonlinear quadratic Volterra integral equations. Computers \& Mathematics with Applications, 62(6), 2555-2566, 2011

[13] R. de Sousa and F. Minhós. On coupled systems of Hammerstein integral equations. Boundary Value Problems, (2019); 2019(7). https://doi.org/10.1186/s13661-019-1122-3.

[14] R. de Sousa and F. Minhós. Coupled systems of Hammerstein-type integral equations with sign-changing kernels. Nonlinear Analysis: Real World Applications, 50, 469-483, 2019

[15] G. Adomian. A review of the decomposition method in applied mathematics. Journal of Mathematical Analysis and Applications, 135(2), 501-544, 1988.

[16] L. Wu, L. Xie and J. Zhang. Adomian decomposition method for nonlinear differential-difference equations. Communications in Nonlinear Science and Numerical Simulation, 14(1):12-18, 2009.

[17] J. H. Kuang and C. J. Chen. Adomian decomposition method used for solving nonlinear pull-in behavior in electrostatic micro-actuators. Mathematical and Computer Modelling, 41(13), 1479-1491, 2005.

[18] E. H. Aly, A. Ebaid and R. Rach. Advances in the Adomian decomposition method for solving two-point nonlinear boundary value problems with Neumann boundary conditions. Computers \& Mathematics with Applications, 63(6), 1056-1065, 2012.

[19] M. M. Hosseini. Adomian decomposition method with Chebyshev polynomials. Applied Mathematics and Computation, 175(2), 1685-1693, 2006.

[20] A. M. Wazwaz and S. M. El-Sayed. A new modification of the Adomian decomposition method for linear and nonlinear operators. Applied Mathematics and Computation, 122(3), 393-405, 2001.

[21] L. Song and W. Wang. A new improved Adomian decomposition method and its application to fractional differential equations. Applied Mathematical Modelling, 37(3), 1590-1598, 2013.

[22] A. F. Qasim and E. S. AL-Rawi. Adomian decomposition method with modified Bernstein polynomials for solving ordinary and partial differential equations. Journal of Applied Mathematics, (2018); 2018, Article ID 1803107, 9 pages. https://doi.org/10.1155/2018/1803107.

[23] Y. Liu. Adomian decomposition method with orthogonal polynomials: Legendre polynomials. Mathematical and Computer Modelling, 49(1-2), 1268-1273, 2009.

[24] Y. Mahmoudi,M. Abdollahi, N. Karimian and H. Khalili . Adomian decomposition method with Laguerre polynomials for solving ordinary diferential equation. Journal of Basic and Applied Scientific Research, 2(12), 
12236-12241, 2012.

[25] S. Mahmood, R. Shah, H. Khan and M. Arif. Laplace Adomian decomposition method for multi dimensional time fractional model of Navier-Stokes equation. Symmetry, (2019); 11(2), 149. https://doi.org/10.3390/sym11020149.

[26] Y. Daoud and A. A. Khidir. Modified Adomian decomposition method for solving the problem of boundary layer convective heat transfer. Propulsion and Power Research, 7(3), 231-237, 2018.

[27] P. V. Ramana and B. K. Raghu Prasad. Modified Adomian decomposition method for Van der Pol equations. International Journal of Non-Linear Mechanics, 65,121-132, 2014.

[28] O. H. Mohammed and H. A. Salim. Computational methods based laplace decomposition for solving nonlinear system of fractional order differential equations. Alexandria Engineering Journal, 57(4), 3549-3557, 2018.

[29] E. Voronovskaya. Determination de la forme asymptotique d'approximation des fonctions par les polynomes de M. Bernstein. Doklady Akademii Nauk SSSR, 79-85, 1932.

[30] A. M. Wazwaz. Linear and nonlinear integral equations: methods and applications. Springer. Saint Xavier University Chicago, USA, (2011). 